\title{
Quantitative multilayered assessment of skin lightening by photoacoustic microscopy
}

\author{
Zhiyang Wang ${ }^{1,2} \wedge$, Fei Yang ${ }^{1,2}$, Zhongwen Cheng ${ }^{1,2}$, Wuyu Zhang ${ }^{1,2}$, Kedi Xiong ${ }^{1,2}$, Tianding Shen ${ }^{3}$, \\ Sihua Yang ${ }^{1,2}$
}

${ }^{1}$ MOE Key Laboratory of Laser Life Science \& Institute of Laser Life Science, College of Biophotonics, South China Normal University, Guangzhou, China; ${ }^{2}$ Guangdong Provincial Key Laboratory of Laser Life Science, College of Biophotonics, South China Normal University, Guangzhou, China; ${ }^{3}$ Department of Plastic and Aesthetic Surgery, Nanfang Hospital, Southern Medical University, Guangzhou, China

Contributions: (I) Conception and design: S Yang, Z Wang; (II) Administrative support: S Yang, K Xiong; (III) Provision of study materials or patients: S Yang, T Shen; (IV) Collection and assembly of data: Z Wang, F Yang, Z Cheng; (V) Data analysis and interpretation: Z Wang, F Yang, W Zhang; (VI) Manuscript writing: All authors; (VII) Final approval of manuscript: All authors.

Correspondence to: Sihua Yang. College of Biophotonics, South China Normal University, No. 55, Zhongshan Avenue West, Tianhe District, Guangzhou 510631, China. Email: yangsh@scnu.edu.cn.

Background: With the emergence of various new skin-lightening products, there is an urgent need to scientifically evaluate the efficacy and toxicology of these products, and provide scientific guidance for their use based on physiological differences between individuals. Visualized imaging methods and quantitative evaluation criteria play key roles in evaluating the efficacy of skin-lightening products. In order to quantify the changes in the multilayered morphology and endogenous components of human skin before and after the use of lightening products, high-resolution three-dimensional (3D) imaging of human skin is required.

Methods: In this study, photoacoustic microscopy (PAM; SSPM-532, Guangdong Photoacoustic Medical Technology Co., Ltd.) was used to capture the morphological structures of human skin and reveal skin components quantitatively. The efficacy and safety of skin-lightening products were evaluated by measuring skin melanin concentration and observing skin morphology. The melanin concentration in the epidermis was obtained by examining the linear relationship between photoacoustic (PA) signals. Further, the epidermal thickness and the melanin distribution were obtained in the cross-sectional $(x-z)$ and lateral $(x-y)$ images. Finally, the efficacy of skin-lightening products was evaluated according to the concentration and distribution of melanin in the epidermis, and the safety of cosmetics was assessed by observing the vascular morphology in the dermis.

Results: PAM noninvasively could assess the multilayered morphological structures of human skin, which allowed for quantification of epidermal thickness and melanin concentration of different skin sites. Based on this, the efficacy and safety of skin-lightening products in multilayer structures were quantitatively evaluated. Conclusions: As a quantitative imaging method, PAM, has the potential to accurately evaluate the use of skin-lightening products. The method can also be extended to assessments within the larger field of aesthetic medicine.

Keywords: Multilayer; photoacoustic microscopy (PAM); skin lightening

Submitted Mar 25, 2021. Accepted for publication Jul 08, 2021.

doi: $10.21037 /$ qims-21-335

View this article at: https://dx.doi.org/10.21037/qims-21-335

\footnotetext{
^ ORCID: 0000-0002-9262-2899.
} 


\section{Introduction}

Skin pigmentation has often been a prominent aesthetic for many people (1). Skin color is determined by the content and spatial distribution of pigment in the skin. Among these pigments, melanin, produced by melanocytes, is the most significant determinant of skin color.

Many people seek ways to whiten their skin. Skinlightening products have become a common choice because of their convenience and effectiveness. Thus, various new skin-lightening products have been developed to meet this demand, and different compounds are used as lightening agents in new skin-lightening products. Among these compounds, pure chemicals have become commonly used skin-lightening products because of their excellent lightening effects. However, pure chemicals may cause side effects such as irritation (2), allergy (3), and risk of cancer when used over a long period (4). Pure natural plant extracts have been proposed as a feasible alternative to pure chemicals, but their lightening effect is unreliable due to the limitation of the components that make up the pure natural plant extracts. The production of modern skin-lightening products are controlled for a number of reasons. On the one hand, manufacturers, to promote the efficacy of their product, ensure the appropriate amount of lightening agent is added. On the other hand, regulatory authorities prevent the use of harmful ingredients to guarantee user safety (5).

The analysis of specific ingredients is the most common evaluation method of skin-lightening products. However, the differences and complexity of skin structures between individuals makes this method unreliable, and a reference or definitive assessment of lightening efficacy and toxicology cannot be provided. It should also be noted that improper use of skin-lightening products often causes adverse side effects such as skin inflammation and rash (6). Therefore, a safe and reliable method that allows for differentiation between individuals is urgently needed to evaluate the effects of skin-lightening products.

Conventional detection methods, such as the human eye or a colorimeter, can only glean information about the skin's surface. These methods cannot quantitatively measure the distribution of melanin and hemoglobin or epidermal thickness. In order to obtain information from beneath the skin, optical detection methods, such as reflected light spectrometry and melanin absorption spectroscopy, can quantify the content of melanin in the skin and evaluate lightening effects on the skin $(7,8)$. However, these instruments cannot visualize the effects on skin structure or characterize the spatial distribution of melanin in the epidermis. Human skin presents a complex heterogeneous medium and has variable depth distribution of blood and pigment content (9). Skin products are mainly used to inhibit the synthesis of melanin or accelerate the breakdown of melanin, which is mainly distributed throughout the stratum corneum and stratum basale (10). However, the side effects of skin products, such as inflammation and rash, usually occur in the vascular network of the superficial dermis. Visible reflectance spectroscopy can quantify the epidermal melanin content but cannot determine melanin distribution. Therefore, the development of a new detection method would be useful for the noninvasive investigation of the morphological structures of each skin layer and for revealing the spatial distribution of chromophore, which plays an important role in the quantitative assessment of the efficacy and safety of skin-lightening products.

The new detection approach of photoacoustic microscopy (PAM) (11-24) has been proposed and developed in dermatology to noninvasively visualize the epidermal melanin content and dermal microvasculature of human skin (25-36). However, studies that have used PAM lack fine-scale visualization of multilayered skin structures (including of the stratum corneum, stratum basale, and dermis) and the distribution of endogenous pigments. There have been no investigations conducted into the longterm monitoring of multilayered skin structures during skin lightening. Therefore, we extended PAM into the field of skin lightening to capture the morphological structures of human skin, quantitatively evaluate skin components, and provide detailed insights into the skin-lightening efficacy and safety of skin-lightening products. High-resolution visualizations of the anatomical structures of the stratum corneum, stratum basale, and dermis were obtained simultaneously by photoacoustic (PA) cross-sectional $(\mathrm{x}-\mathrm{z})$ and lateral $(x-y)$ imaging of skin layers. The lightening efficacy of skin-lightening products was evaluated by analyzing the stratum corneum and stratum basale, and toxicology and safety were investigated by observing the morphology of microvasculature in the dermis. PAM is a useful method for scientifically evaluating and guiding the use of skin-lightening products.

\section{Methods}

\section{Assessment methods}

The study was conducted in accordance with the 


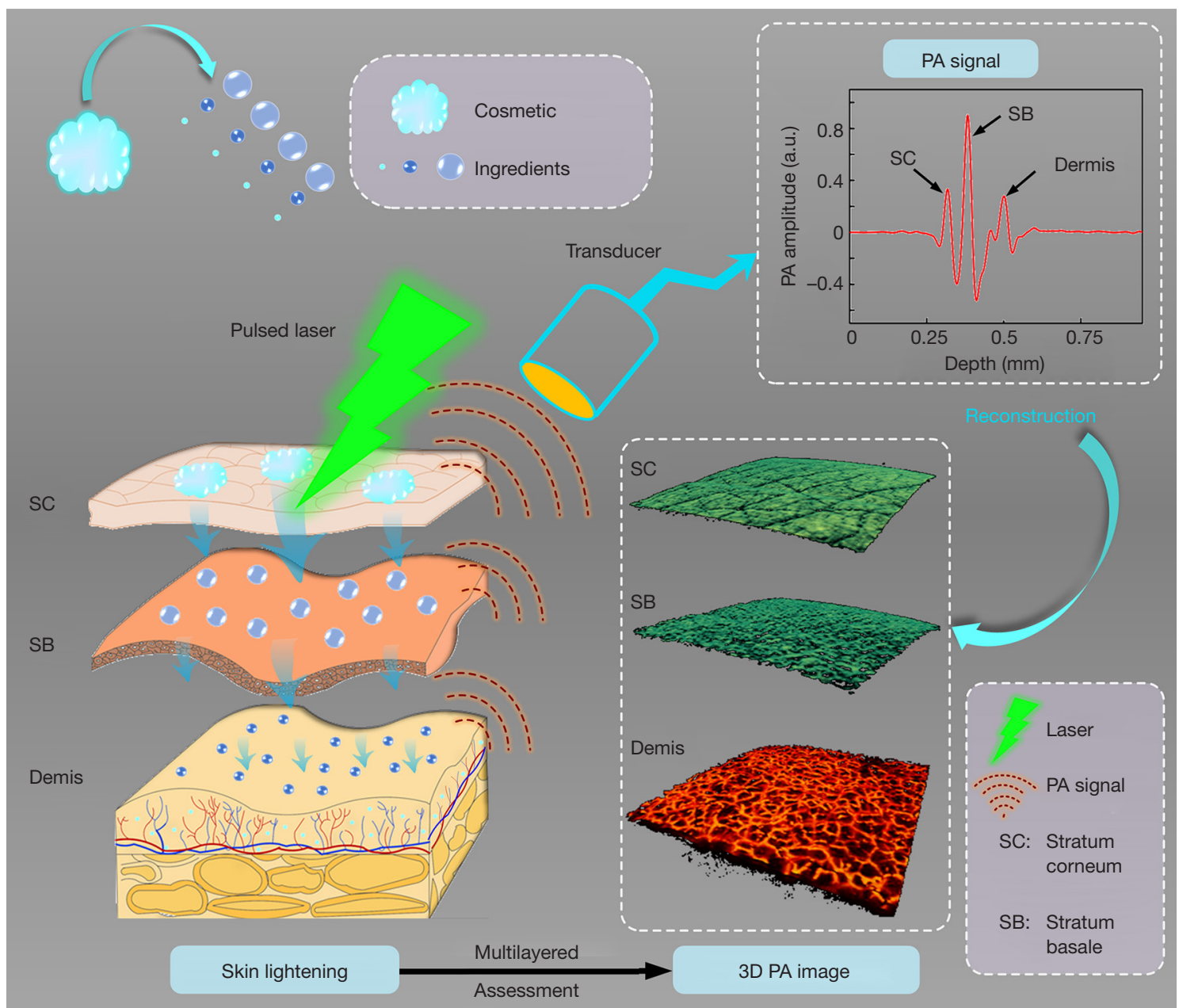

Figure 1 The interaction process of skin-lightening products, and the multilayered 3D reconstruction of skin structures by PAM. 3D, threedimensional; PAM, photoacoustic microscopy; PA, photoacoustic; SC, stratum corneum; SB, stratum basale.

Declaration of Helsinki (as revised in 2013). The study was approved by the Chinese Ethics Committee of Registering Clinical Trials (ChiECRCT20200184) and registered with the Chinese Clinical Trial Registry (ChiCTR2000034400). Before skin imaging, written informed consent was received from all participants. During the monitoring of the skinlightening effect, the chosen skin-lightening products were commercial sunscreen, lightening cream, and lightening toner. The key lightening component was nicotinamide. The concentration of the active component was not disclosed by the manufacturer. The products were used in the morning and evening as per their instructions. The volunteers consistently used the products for 1 month. PA imaging was performed on the first, tenth, twentieth, and thirtieth days. A schematic illustration of multilayered assessment of skin lightening using PAM is shown in Figure 1, which graphically illustrates the process of skin lightening and multilayered assessment of the efficacy of skin-lightening products through three-dimensional (3D) PA imaging. After the lightening products are applied to the skin, the ingredients of the products begin to act on the epidermis of the skin to inhibit the synthesis of melanin and accelerate the discharge. Then, the action area is investigated by using a PAM. First, the pulsed laser irradiates the skin to generate PA signals. The PA signals containing depth information are received by the ultrasound transducer to reconstruct multilayered skin structures. The structures of the different skin layers are distinguished by Aline signals. The first signal peak represents the stratum corneum in the epidermis. The amplitude of the 
second peak is the highest, which represents the strong absorption of melanin in the stratum basale. The posterior absorption peaks represent the blood vessels in the dermis. The thickness and location of these skin structures can be calculated and separated based on the sampling rate and the sound velocity. In summary, PAM can provide a useful microscopic imaging method in quantitatively assessing skin structures.

\section{Imaging system and parameters}

The schematic diagram of the PAM system is shown in Figure $2 A$. A pulsed laser operating at a wavelength of $532 \mathrm{~nm}$ with $\sim 7 \mathrm{~ns}$ duration and a repetition rate of $10 \mathrm{kHz}$ was used as the excitation source. To improve the lateral resolution, the laser beam was first cleaned using a pinhole and then coupled to a single-mode fiber (460 HP, Thorlabs, USA) by an objective lens ( $4 \times$, Daheng Optics, China). The single-mode fiber guided the excitation laser to the integrated scanning probe. The internal excitation laser path of the integrated probe is shown in Figure 2B. The laser beam was collimated by a fiber collimator (F240FC-532, Thorlabs, NA $=0.51$ ) and then focused by an objective lens to illuminate the skin tissue. Below the integrated scanning head, a coupling cup filled with deionized water was used against the skin to couple PA signals. The deionized water came into contact with the skin through a transparent and biocompatible membrane. The high-frequency ultrasonic transducer with a focal length of $8 \mathrm{~mm}$ and a $-6 \mathrm{~dB}$ bandwidth of $110 \%$ was used to receive the PAM signals. The piezoelectric element (Piezotech, France) self-focused through a bowl-shaped structure. A two-dimensional (2D) scanner (LS2-25T, Jiancheng Optics, China) was used for volume imaging. The acoustic focus and optical focus were confocal and scanned pixel by pixel to obtain high-resolution images. The PA signals were amplified (LNA-650, RF Bay, USA) and digitized with a data acquisition card (M3i.3221, Spectrum, Germany) at a sampling rate of $250 \mathrm{MS} / \mathrm{s}$. The acquired data were restored and reconstructed in real time based on the Laboratory Virtual Instrument Engineering Workbench (v. 2016, LabVIEW, National Instruments, USA). The chosen LabVIEW program included scan control, data acquisition, data storage, and image reconstruction functions. To demonstrate the microscopic imaging parameters of the system, we investigated the spatial resolution of the PAM system. A sharp-edged surgical blade was imaged, as shown in Figure 2C. The edge-spread function (red line) was calculated by taking the distribution of PA normalized function values along the white line in PA images. Taking the derivative of the red line yielded the linespread function (blue line). Hence, the lateral resolution of the system as defined by the full-width at half-maximum (FWHM) of the blue line was $6.4 \mu \mathrm{m}$. The envelope of the PA Aline signal (Figure 2D) indicated that the axial resolution was $25.3 \mu \mathrm{m}$. Figure $2 E$ shows the absorption coefficient spectra of endogenous tissue chromophores in the human skin (37), which indicated that both melanin and hemoglobin had high absorption at $532 \mathrm{~nm}$. To characterize the relationship between PA signal amplitude and concentration of melanin, the PAM amplitudes of different concentrations of melanin solutions were measured using the PAM system, and then the peak-to-peak values of the PA Aline signal were extracted. At last, the linear relationship was determined, as shown in Figure $2 F$, so the concentration of melanin could be quantitatively calculated by the determination coefficient (R2).

\section{Results}

\section{In vivo imaging of the opisthenar}

The investigations illustrated in Figure 3 were performed to demonstrate the ability of visualizing multilayered skin structures. The pulsed laser with illumination energy of $12 \mathrm{~mJ} / \mathrm{cm}^{2}$ was less than the American National Standard for Safe Use of Lasers (ANSI) safety limit (38). To better match the PA images with the anatomical structures of the skin, a typical image was created with hematoxylin and eosin (HE) histopathology of the human skin (Figure 3A). The epidermis and dermis could be clearly distinguished in the HE-stained image. The stratum corneum and stratum basale of the epidermis contained a large amount of melanin, while the chromophores in the dermis were mainly hemoglobin in the blood vessels. For PA data treatment, the $3 \mathrm{D}$ volume PA data were obtained in an imaging experiment. First, the skin morphology was observed to obtain skin profiles (white dashed lines as seen in Figure 3B) through the $\mathrm{x}-\mathrm{z}$ cross-sectional images. Then, according to the skin profiles and thickness of the stratum corneum and stratum basale, all $\mathrm{x}-\mathrm{z}$ cross-sectional images were segmented according to their depth to obtain the stratum corneum, stratum basale, and dermis. Figure $3 B$ shows a PA cross-sectional image of human skin. The distribution of depth of chromophores was clearly visualized in the PA cross-sectional image, which was mostly consistent with the HE-stained image. The results show that PA imaging noninvasively obtained 
A

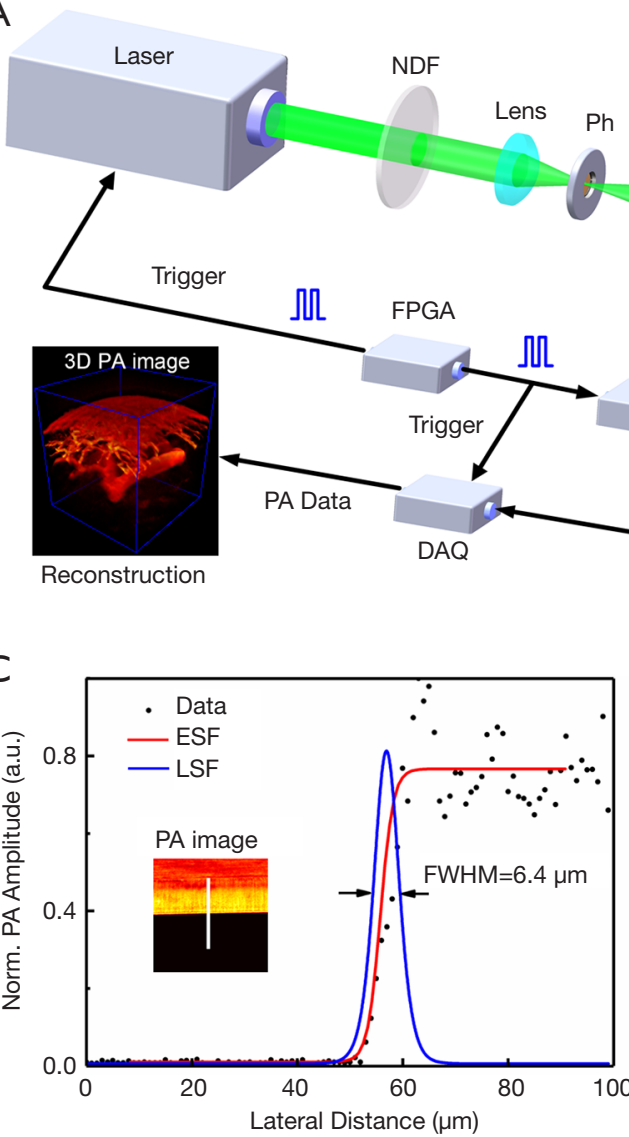

$E$

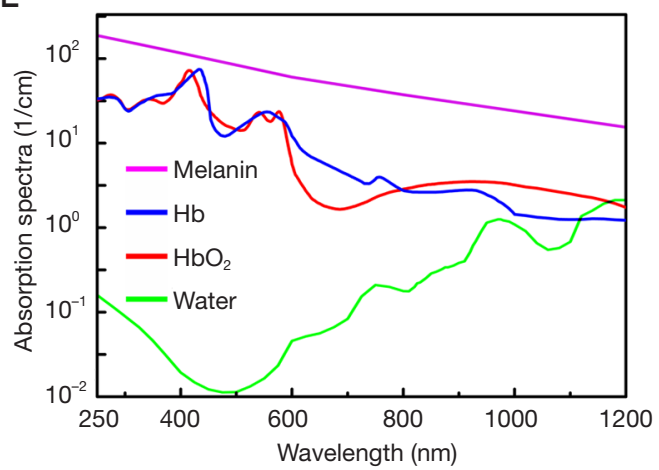

B

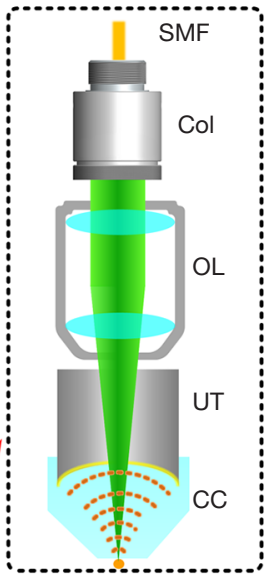

$\mathrm{D}$

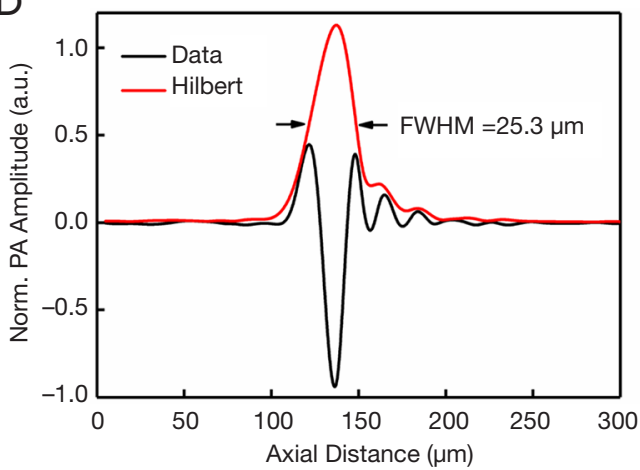

$\mathrm{F}$

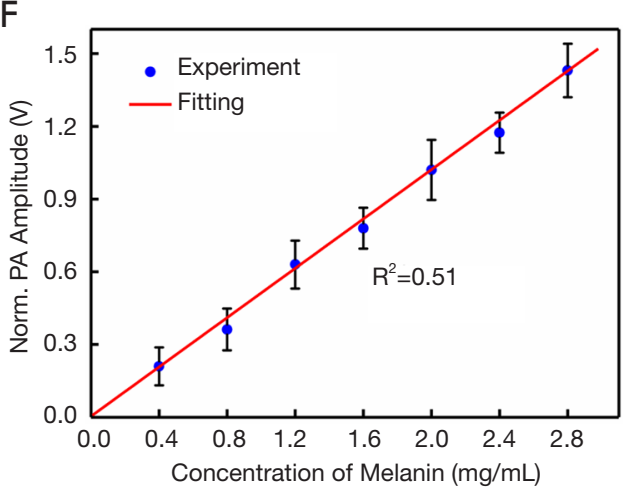

Figure 2 Schematic diagram and imaging parameters of the PAM system. (A) Schematic diagram of the PAM system. (B) The internal excitation laser path of the integrated probe. (C) The measured lateral resolution of PA imaging by sharp-edged blade method. (D) The measured axial resolution of PA imaging. (E) Absorption spectra of endogenous absorbers in human skin. (F) The linear relationship between PA signal amplitude and concentration of melanin. PAM, photoacoustic microscopy; PA, photoacoustic; NDF, neutral density filter; $\mathrm{Ph}$, pinhole; OL, objective lens; SMF, single-mode fiber; FPGA, field programmable gate array; DAQ, data acquisition; Col, collimator; UT, ultrasonic transducer; CC, coupling cup; ESF, edge-spread function; LSF, line-spread function; FWHM, full-width at half-maximum; Hb, hemoglobin; $\mathrm{HbO}_{2}$, oxyhemoglobin. 

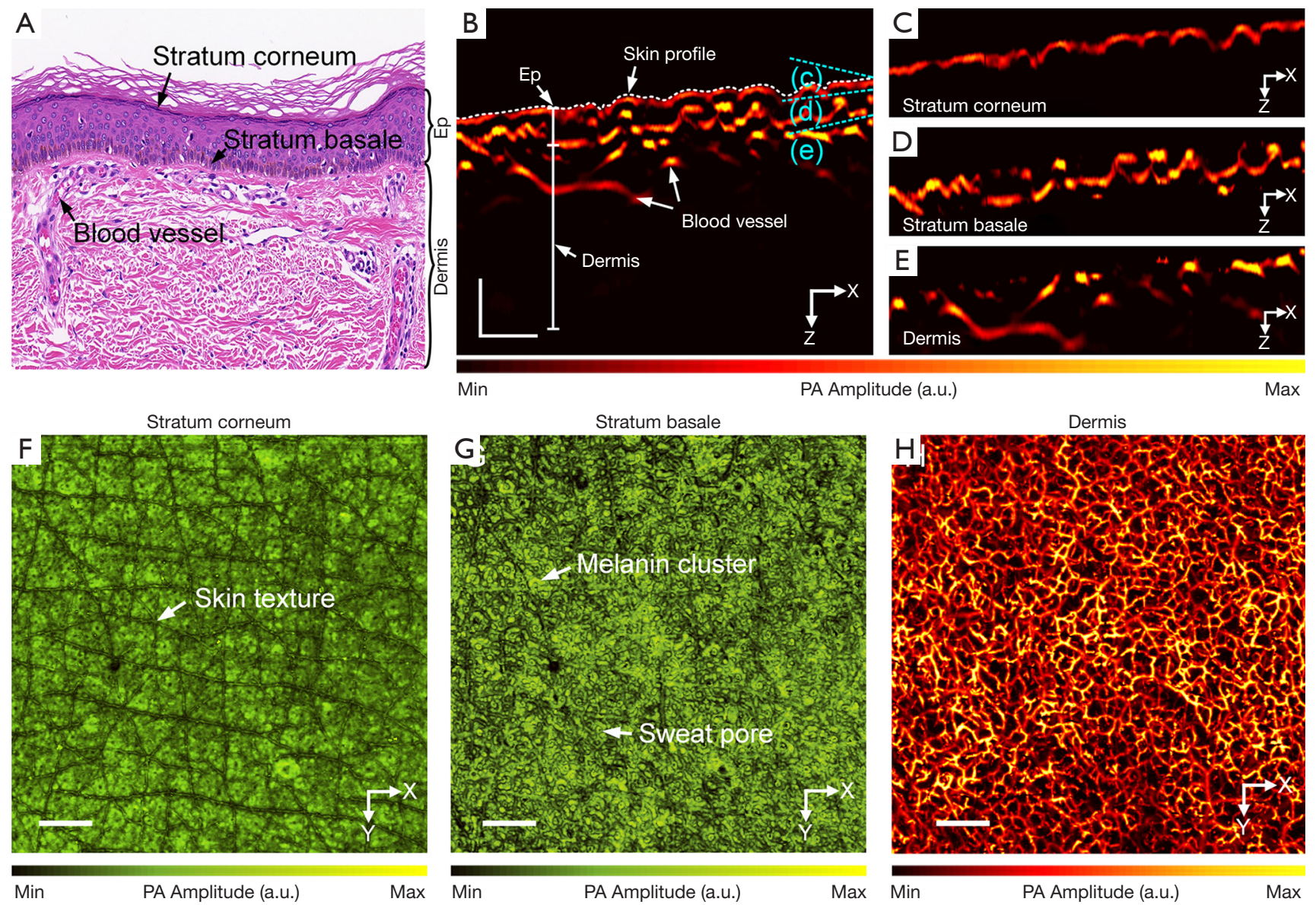

Figure 3 In vivo imaging of the opisthenar. (A) A typical skin image with HE histopathology, the image magnified by a factor of 33. (B) The PA cross-sectional image of human skin. (C,D,E) The separated PA cross-sectional images of the stratum corneum, stratum basale, and dermis layers. (F,G,H) PA lateral MAP images of the stratum corneum, stratum basale, and dermis layer. Scale bar: $1 \mathrm{~mm}$. HE, hematoxylin and eosin; PA, photoacoustic; MAP, maximum amplitude projection; Ep, epidermis.

the morphological structures of human skin. Figure $3 C-3 E$ respectively represent the PA cross-sectional images of the stratum corneum, stratum basale, and dermis. Furthermore, the lateral maximum amplitude projections (MAPs) of the stratum corneum, stratum basale, and dermis are shown in Figure $3 \mathrm{~F}-3 \mathrm{H}$, which clearly demonstrate morphological structures in different skin layers. Figure $3 F$ shows a highresolution image of skin texture traits of the stratum corneum. The melanin clusters and pores in the stratum basale are shown in Figure 3G. The cross-sectional and MAP images provide important information for evaluating the efficacy of skin-lightening products. Figure $3 \mathrm{H}$ shows the abundant vascular networks in the dermis, and the branching and morphology of these microvasculature are shown to be closely related to the physiological condition of the skin. Therefore, PA imaging could effectively evaluate the safety of the products by monitoring the dermis. The imaging experiments demonstrated the potential for the PAM system to noninvasively evaluate the efficacy and safety of skin-lightening products.

\section{In vivo imaging of different skin sites}

To further confirm the feasibility of the PAM system, images of four different skin sites were obtained to visualize different skin structures (Figure 4). These areas were chosen because they are sites where skin-lightening products are often used. Figure $4 A-4 D$ show the PA lateral MAP images of the epidermal layer of the opisthenar, lateral forearm, inner forearm, and cheek, respectively. The structure of the 

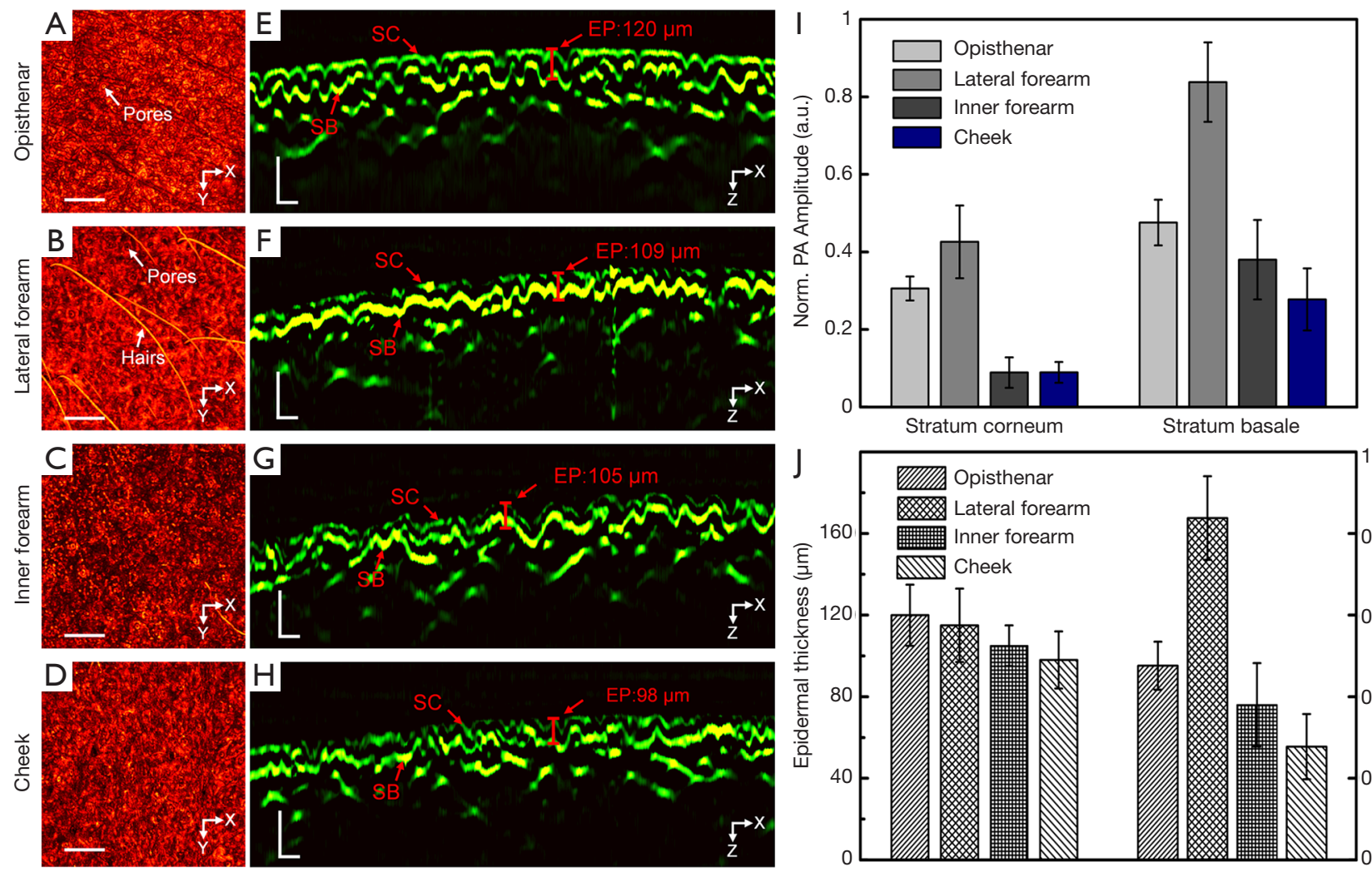

Min PA Amplitude (a.u.)Max Min

PA Amplitude (a.u.)

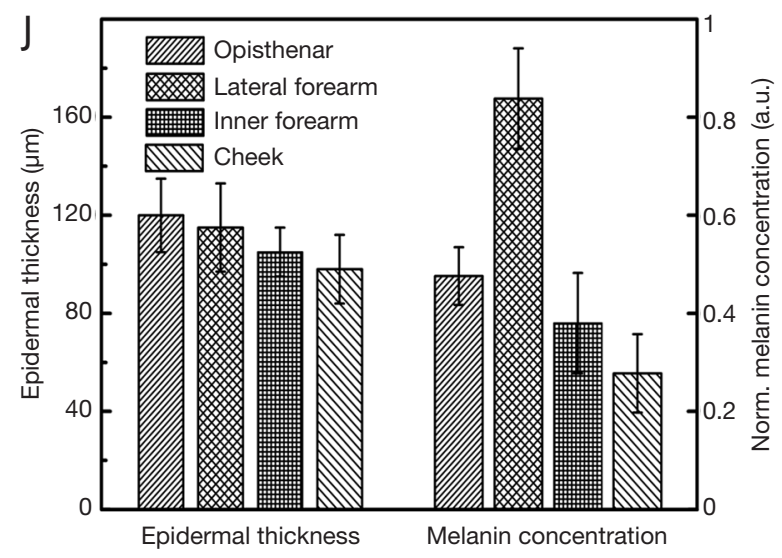

Figure 4 In vivo imaging of different skin sites. (A-D) PA lateral MAP images of the opisthenar, lateral forearm, inner forearm, and cheek in the epidermis. Scale bar: $1 \mathrm{~mm}$. (E-H) The PA cross-sectional images of the opisthenar, lateral forearm, inner forearm, and cheek. Scale bar: $0.2 \mathrm{~mm}$. (I) The statistics of PA amplitude of the stratum corneum and stratum basale at different imaging sites. (J) Epidermal thickness and melanin concentration at different imaging sites. MAP, maximum amplitude projection; PA, photoacoustic; SC, stratum corneum; SB, stratum basale; Ep, epidermis.

epidermis at different imaging sites show variable features in texture. Skin texture and pores were clearly observed on the opisthenar, and the lateral forearm exhibited large pores and hair. The skin on the inner forearm and the cheek was relatively fine and smooth, with shallow texture features. Figure $4 E-4 H$ show the PA cross-sectional images of different skin sites. The stratum corneum of the cheek was thin, which was helpful for melanin breakdown. In the PA cross-sectional images, the thickness and absorption intensity of the stratum corneum and stratum basale were quantitatively evaluated. Figure $4 I$ shows the PA signal amplitude of the stratum corneum and stratum basale at different imaging sites. There was a linear relationship between the PA signal amplitude and the concentration of melanin, so the specific concentration of melanin was calculated using the PA signal amplitude. As shown in
Figure 47, the epidermal thickness of the lateral forearm was approximately $109 \mu \mathrm{m}$, which was also the highest melanin concentration, while the facial epidermis was only $98 \mu \mathrm{m}$, which was the lowest melanin concentration. The imaging results demonstrate that the PAM system revealed different skin structures and quantitatively measured skin structures and components.

\section{In vivo monitoring of the skin-lightening effect}

We monitored the effect of skin-lightening products and moisturizing products on the skin over 30 days. Figure 5A-5D show the PA cross-sectional images and the lateral MAP images on the first, tenth, twentieth, and thirtieth days. In this experiment, to ensure that the same imaging area was analyzed, a nevus was set as a 

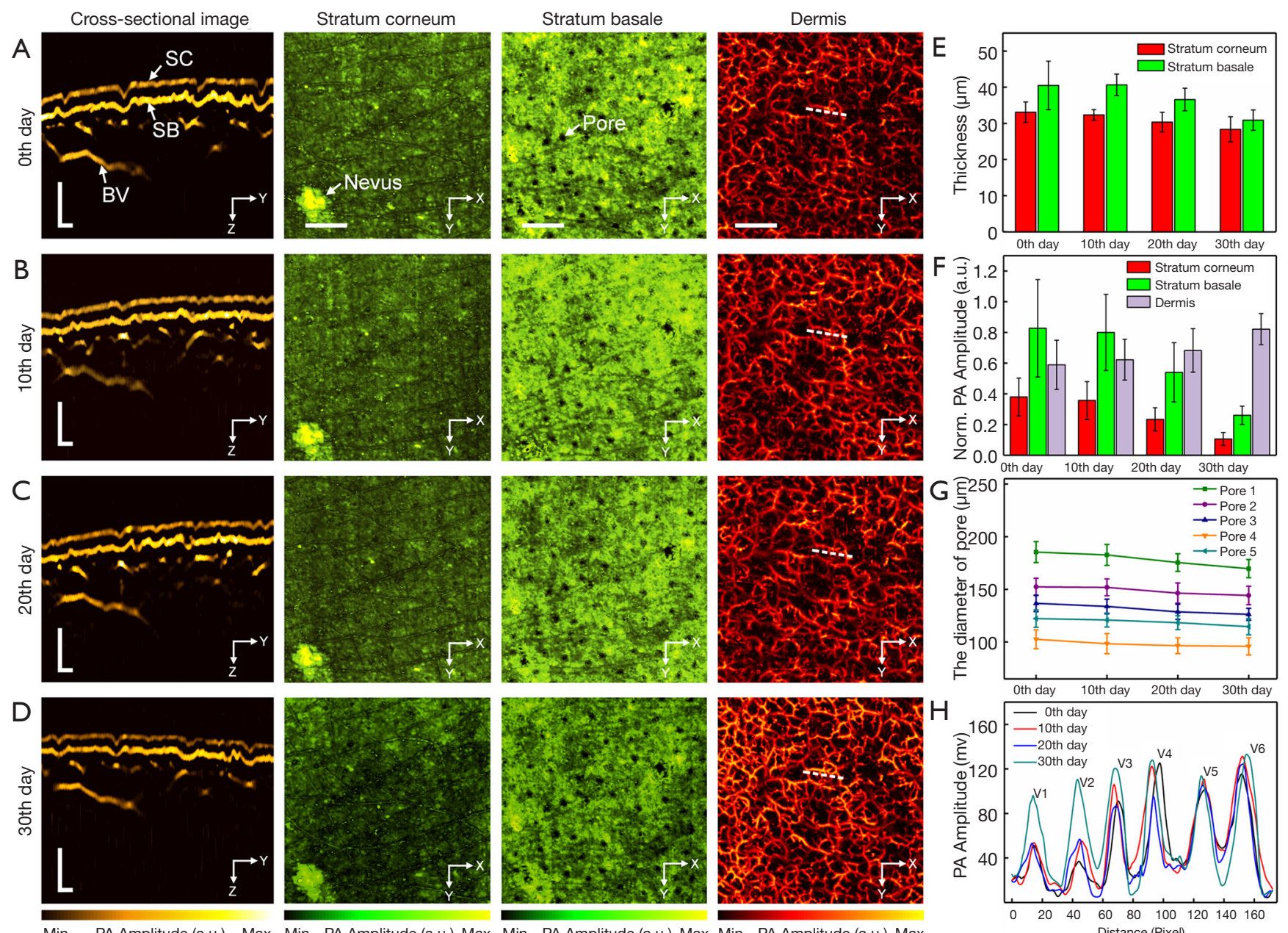

Min PA Amplitude (a.u.) Max Min PA Amplitude (a.u.) Max Min PA Amplitude (a.u.) Max Min PA Amplitude (a.u.) Max

Figure 5 In vivo monitoring of the skin lightening effect. (A-D) The PA cross-sectional images and lateral MAP images of the stratum corneum, stratum basale, and dermis on the first, tenth, twentieth, and thirtieth days after using skin-lightening products. Scale bar in crosssectional (y-z) images: $0.2 \mathrm{~mm}$; scale bar in lateral $(x-y)$ images: $1 \mathrm{~mm}$. (E) The statistical thickness of the stratum corneum and stratum basale on the first, tenth, twentieth, and thirtieth days. (F) The statistical PA signal amplitude of the stratum corneum, stratum basale, and dermis on the first, tenth, twentieth, and thirtieth days. (G) The pore diameters in the stratum basale on the first, tenth, twentieth, and thirtieth days. $(\mathrm{H})$ The curves of PA signal amplitudes corresponding to the white dashed lines marked on the dermis. PA, photoacoustic; MAP, maximum amplitude projection; SC, stratum corneum; SB, stratum basale; BV, blood vessel.

reference point. The nevus was used to assist in marking and capturing the same imaging area. PA images show that although the skin surfaces and coupling cup were inconsistent in each imaging experiment and rendered the skin surface tilt inconsistent, there was no other obvious distortion of the morphological structures. The depth distribution and thickness of each structure were obtained from the cross-sectional images, and the entire lateral morphological structures of each layer were observed in MAP images. Five sites were randomly selected to analyze the thickness and the PA signal amplitude (Figure 5E, 5F). Mean value and standard deviation were calculated, and the error bars in Figure 5E, $5 F$ represent the standard deviation. As shown in Figure 5E-5H, we quantitatively analyzed the efficacy and safety of the use of skin-lightening products for 30 days on multilayered structures. Figure $5 E$ shows that there was no obvious change in the thickness of the stratum corneum or stratum basale on the tenth day, but there was a slight decrease in the thickness on the twentieth and thirtieth days. These findings showed that long-term use of 
skin-lightening products changed melanin distribution and thickness of the epidermis. Figure $5 F$ shows the PA signal amplitude of the stratum corneum and stratum basale, indicating that the absorption intensity was significantly decreased on the thirtieth day. Figure $5 G$ shows changes in the diameters of five pores in the stratum basale on the first, tenth, twentieth, and thirtieth days. The results show that the use of moisturizing products decreased the diameters of pores, indicating that the skin-lightening products improved the skin condition.

In order to verify the safety of the skin-lightening products, we analyzed the vascular structures, as shown in Figure 5H. A detailed comparison of the profiles taken along the white dashed lines in the dermis show that the vascular morphology of the dermis was mostly consistent on the first, tenth, twentieth, and thirtieth days. Under normal physiological conditions, vascular morphology changes slowly in the dermis. In contrast, when the skin is irritated or inflamed, microvasculature is damaged and vascular morphology changes. In this experiment, the stable vascular morphology proved that the skin-lightening product worked effectively for the volunteer.

\section{Discussion}

Compared with conventional optical detection methods, PAM can deeply penetrate the morphological structures of the skin for high resolution visualization and analysis, without the need for labels. PAM was proposed to evaluate the effect of skin-lightening products on multilayered structures of the skin, taking advantage of the intrinsic optical properties of endogenous chromophores. We investigated the stratum corneum and stratum basale to monitor the efficacy of skin-lightening products and analyzed the safety characteristics of skin-lightening products by observing the vascular morphology of the dermis.

Despite these encouraging findings, the performance of PAM needs to be enhanced by addressing the following limitations before it can be considered for use in clinical evaluation. The first limitation of this study was the use of a $532 \mathrm{~nm}$ laser, which did not allow for the analysis of specific chromophores. In future studies, specific skin chromophores (such as oxyhemoglobin, deoxyhemoglobin, or melanin) can be accurately resolved based on multispectral PA signals and absorption coefficients. In addition, skin atrophy and the thicknesses of the stratum corneum and stratum basale might not have been accurate when the thicknesses were close to the axial resolution of PAM. To improve the axial resolution of the PAM system, higher frequency ultrasonic transducers can be used to more accurately obtain the thickness of each skin structure layer. Finally, the current imaging speed requires approximately $6 \mathrm{~min}$ to cover an area of $10 \mathrm{~mm} \times 10 \mathrm{~mm}$. Breathing or trembling during scanning can degrade image quality, resulting in changes to pathophysiological observations. To eliminate the abovementioned effects of breathing or trembling, a motion correction algorithm is used to eliminate motion artifacts and ensure accurate structural images for use in diagnosis (39). In future, a faster scanner, such as a voice-coil scanner or a micro-electro-mechanical-system-based scanner, would be beneficial in eliminating the effects of motion and improving clinical evaluation.

\section{Acknowledgments}

Funding: This work was supported by the National Natural Science Foundation of China (No. 61822505, 11774101, and 61627827), the Science and Technology Planning Project of Guangdong Province, China (No. 2015B020233016), the Science and Technology Program of Guangzhou (No. 2019050001), and the Special Funds for the Cultivation of Guangdong College Students' Scientific and Technological Innovation ("Climbing Program" Special Funds; No. pdjh2021a0128).

\section{Footnote}

Conflicts of Interest: All authors have completed the ICMJE uniform disclosure form (available at https://dx.doi. org/10.21037/qims-21-335). The authors have no conflicts of interest to declare.

Ethical Statement: The authors are accountable for all aspects of the work in ensuring that questions related to the accuracy or integrity of any part of the work are appropriately investigated and resolved. The study was conducted in accordance with the Declaration of Helsinki (as revised in 2013). The study was approved by the Chinese Ethics Committee of Registering Clinical Trials (ChiECRCT20200184) and registered with Chinese Clinical Trial Registry (ChiCTR2000034400). Before skin imaging, written informed consent was taken from all participants.

Open Access Statement: This is an Open Access article 
distributed in accordance with the Creative Commons Attribution-NonCommercial-NoDerivs 4.0 International License (CC BY-NC-ND 4.0), which permits the noncommercial replication and distribution of the article with the strict proviso that no changes or edits are made and the original work is properly cited (including links to both the formal publication through the relevant DOI and the license). See: https://creativecommons.org/licenses/by-nc-nd/4.0/.

\section{References}

1. Pawaskar MD, Parikh P, Markowski T, McMichael AJ, Feldman SR, Balkrishnan R. Melasma and its impact on health-related quality of life in Hispanic women. J Dermatolog Treat 2007;18:5-9.

2. Camarasa JG, Serra-Baldrich E. Exogenous ochronosis with allergic contact dermatitis from hydroquinone. Contact Dermatitis 1994;31:57-8.

3. Mahé A, Ly F, Aymard G, Dangou JM. Skin diseases associated with the cosmetic use of bleaching products in women from Dakar, Senegal. Br J Dermatol 2003;148:493-500.

4. Kooyers TJ, Westerhof $W$. Toxicology and health risks of hydroquinone in skin lightening formulations. J Eur Acad Dermatol Venereol 2006;20:777-80.

5. Chisvert A, Sisternes J, Balaguer A, Salvador A. A gas chromatography-mass spectrometric method to determine skin-whitening agents in cosmetic products. Talanta 2010;81:530-6.

6. Sautebin L. Understanding the adverse effects of cosmetics: a pilot project in cosmetovigilance. Drug Saf 2008;31:433-6.

7. Zonios G, Bykowski J, Kollias N. Skin melanin, hemoglobin, and light scattering properties can be quantitatively assessed in vivo using diffuse reflectance spectroscopy. J Invest Dermatol 2001;117:1452-7.

8. Zonios G, Dimou A, Bassukas I, Galaris D, Tsolakidis A, Kaxiras E. Melanin absorption spectroscopy: new method for noninvasive skin investigation and melanoma detection. J Biomed Opt 2008;13:014017.

9. Bashkatov N, Genina EA, Kochubey VI, Tuchin VV. Optical properties of human skin, subcutaneous and mucous tissues in the wavelength range from 400 to 2000 nm. J Phys D Appl Phys 2005;38:2543.

10. Young AR. Chromophores in human skin. Phys Med Biol 1997;42:789-802.

11. Zhang HF, Maslov K, Stoica G, Wang LV. Functional photoacoustic microscopy for high-resolution and noninvasive in vivo imaging. Nat Biotechnol 2006;24:848-51.

12. Gao D, Liu Y, Wang Y, Yuan Z. Protein-modified ultrasmall gold clusters for dual-modal in vivo fluorescence/ photoacoustic imaging. Quant Imaging Med Surg 2018;8:326-32.

13. Oraevsky AA, Clingman B, Zalev J, Stavros AT, Yang WT, Parikh JR. Clinical optoacoustic imaging combined with ultrasound for coregistered functional and anatomical mapping of breast tumors. Photoacoustics 2018;12:30-45.

14. Qiu T, Lan Y, Gao W, Zhou M, Liu S, Huang W, Zeng S, Pathak JL, Yang B, Zhang J. Photoacoustic imaging as a highly efficient and precise imaging strategy for the evaluation of brain diseases. Quant Imaging Med Surg 2021;11:2169-86.

15. Shi J, Tang Y, Yao J. Advances in super-resolution photoacoustic imaging. Quant Imaging Med Surg 2018;8:724-32.

16. Wang Y, Li C, Wang RK. Noncontact photoacoustic imaging achieved by using a low-coherence interferometer as the acoustic detector. Opt Lett 2011;36:3975-7.

17. Rao B, Leng X, Zeng Y, Lin Y, Chen R, Zhou Q, Hagemann AR, Kuroki LM, McCourt CK, Mutch DG, Powell MA, Hagemann IS, Zhu Q. Optical resolution photoacoustic microscopy of ovary and fallopian tube. Sci Rep 2019;9:14306.

18. Guo Z, Li Y, Chen SL. Miniature probe for in vivo optical- and acoustic-resolution photoacoustic microscopy. Opt Lett 2018;43:1119-22.

19. Chen J, Zhang Y, Li X, Zhu J, Li D, Li S, Lee C, Wang L. Confocal visible/NIR photoacoustic microscopy of tumors with structural, functional, and nanoprobe contrasts. Photonics Res 2020;8:1875-80.

20. Qin W, Jin T, Guo H, Xi L. Large-field-of-view optical resolution photoacoustic microscopy. Opt Express 2018;26:4271-8.

21. Yang F, Wang Z, Zhang W, Ma H, Cheng Z, Gu Y, Qiu $\mathrm{H}$, Yang S. Wide-field monitoring and real-time local recording of microvascular networks on small animals with a dual-raster-scanned photoacoustic microscope. J Biophotonics 2020;13:e202000022.

22. Liu C, Liao J, Chen L, Chen J, Ding R, Gong X, Cui C, Pang Z, Zheng W, Song L. The integrated high-resolution reflection-mode photoacoustic and fluorescence confocal microscopy. Photoacoustics 2019;14:12-8.

23. He G, Li B, Yang S. In vivo imaging of a single erythrocyte with high-resolution photoacoustic microscopy. Front Optoelectron 2015;8:122-7. 
24. Wang Z, Yang F, Cheng Z, Zhang W, Xiong K, Yang S. Photoacoustic-guided photothermal therapy by mapping of tumor microvasculature and nanoparticle. Nanophotonics 2021. [Epub ahead of print]. doi: 10.1515/ nanoph-2021-0198.

25. Ma H, Yang S, Cheng Z, Xing D. Photoacoustic confocal dermoscope with a waterless coupling and impedance matching opto-sono probe. Opt Lett 2017;42:2342-5.

26. Baik JW, Kim JY, Cho S, Choi S, Kim J, Kim C. Super wide-field photoacoustic microscopy of animals and humans in vivo. IEEE Trans Med Imaging 2020;39:975-84.

27. Ma H, Cheng Z, Wang Z, Zhang W, Yang S. Switchable optical and acoustic resolution photoacoustic dermoscope dedicated into in vivo biopsy-like of human skin. Appl Phys Lett 2020;116:073703.

28. Attia ABE, Balasundaram G, Moothanchery M, Dinish US, Bi R, Ntziachristos V, Olivo M. A review of clinical photoacoustic imaging: current and future trends. Photoacoustics 2019;16:100144.

29. Wang Z, Yang F, Ma H, Cheng Z, Yang S. Photoacoustic and ultrasound (PAUS) dermoscope with high sensitivity and penetration depth by using a bimorph transducer. J Biophotonics 2020;13:e202000145.

30. Aguirre J, Schwarz M, Garzorz N, Omar M, Buehler A, Eyerich K, Ntziachristos V. Precision assessment of label-free psoriasis biomarkers with ultra-broadband optoacoustic mesoscopy. Nat Biomed Eng 2017;1:1-8.

31. Xu D, Yang S, Wang Y, Gu Y, Xing D. Noninvasive and high-resolving photoacoustic dermoscopy of human skin. Biomed Opt Express 2016;7:2095-102.

32. Cheng Z, Ma H, Wang Z, Yang S. In vivo volumetric monitoring of revascularization of traumatized skin using extended depth-of-field photoacoustic microscopy. Front Optoelectron 2020;13:307-17.

33. Hariri A, Moore C, Mantri Y, Jokerst JV. Photoacoustic imaging as a tool for assessing hair follicular organization. Sensors (Basel) 2020;20:5848.

34. Watanabe T, Tamura A, Yoshimura Y, Nakazawa H. Determination of melanin in human hair by photoacoustic spectroscopy. Anal Biochem 1997;254:267-71.

35. Favazza CP, Jassim O, Cornelius LA, Wang LV. In vivo photoacoustic microscopy of human cutaneous microvasculature and a nevus. J Biomed Opt 2011;16:016015.

36. Viator JA, Komadina J, Svaasand LO, Aguilar G, Choi B, Stuart Nelson J. A comparative study of photoacoustic and reflectance methods for determination of epidermal melanin content. J Invest Dermatol 2004;122:1432-9.

37. Beard P. Biomedical photoacoustic imaging. Interface Focus 2011;1:602-31.

38. American National Standard for Safe Use of Lasers. Laser Institute of America, 2014: ANSI Z136.1-2014.

39. Cheng Z, Ma H, Wang Z, Zhang W, Yang F, Yang S. Subpixel and on-line motion correction for photoacoustic dermoscopy. IEEE J Sel Top Quant 2020;27:1-8.
Cite this article as: Wang Z, Yang F, Cheng Z, Zhang W, Xiong K, Shen T, Yang S. Quantitative multilayered assessment of skin lightening by photoacoustic microscopy. Quant Imaging Med Surg 2022;12(1):470-480. doi: 10.21037/qims-21-335 